\title{
Análise de Contexto em Interações com o SCALA Tablet Mediando a Comunicação de Alunos Incluídos com Autismo
}

\author{
Analysis on Interactions With SCALA Tablet Facilitating \\ Communication for Students With Autism Included
}

\begin{abstract}
Resumo: Uma das práticas culturais mais significativas dos seres humanos é a comunicação desenvolvida na interação com os outros num processo sócio-histórico. Este artigo apresenta um recorte do projeto SCALA (Sistema de Comunicação Alternativa para Letramento de crianças com Autismo) que foca no desenvolvimento de um sistema de comunicação alternativa em duas versões (web e android) e tem como finalidade facilitar a comunicação e a interação de sujeitos com autismo que apresentam déficits. Neste estudo, discute-se aspectos referentes a uma análise do contexto onde ocorrem as interações entre os sujeitos a partir de coleta de dados com duas crianças entre 3 e 5 anos, com Transtorno do Espectro Autista, não oralizados e incluídos na educação infantil. Os resultados permitem compreender o potencial de um sistema de comunicação para proporcionar possibilidades de inclusão destas crianças, no contexto da educação infantil, com aumento da comunicação e interação com o uso do Sistema SCALA.
\end{abstract}

Palavras-chave: Transtorno do Espectro Autista. Autismo. Comunicação Alternativa. Contexto. Tablet.

Abstract: Communication is one of the most significant cultural practices of human beings and it's developed in interaction with others in a social-historical process. This article presents part of the SCALA project (Alternative Communication System for Literacy for children with Autism) that focuses on developing an alternative communication system in two versions (web and android), that aims to facilitate communication and interaction of individuals with autism that have deficits. In this study, we discuss aspects related to an analysis of the context where occurring the interactions between subjects. Data collection was made with two children between 3 and 5 years with Autism Spectrum Disorder, not oralized and enrolled in early childhood education. The results allow to understand the potential of a communication system to provide opportunities of inclusion of these children, in the context of early childhood education, with increased communication and interaction using the SCALA system.

Keywords: Autism Spectrum Disorder. Autism. Alternative Communication. Context. Tablet.

COUTINHO, Katia Soares; BEZ, Maria Rosangela; PASSERINO, Liliana Maria. Análise de contexto em interações com o SCALA Tablet mediando a comunicação de alunos incluídos com autismo. Informática na Educação: teoria e prática, Porto Alegre, v. 17, n. 1, p. 221-231, jan./jun. 2014.

\author{
Katia Soares Coutinho \\ Hospital de Clínicas de Porto Alegre
}

Maria Rosangela Bez

Universidade Federal do Rio Grande do Sul

Liliana Maria Passerino

Universidade Federal do Rio Grande do Sul

\section{Introdução}

A comunicação humana é uma das mais significativas práticas culturais da humanidade. $O$ envolvimento dos sujeitos em situações comunicativas não é inato nem depende da maturação orgânica, mas sim um processo sócio-histórico que se desenvolve ao longo da vida. As formas mais comuns de comunicação são a oral e a escrita, mas o processo comunicacional inclui também expressões gestuais e corporais, bem como envolve também aspectos relativos à intersubjetividade, como a reciprocidade e as crenças dos sujeitos em interação. O processo de apropriação da linguagem é extremamente relevante no desenvolvimento 
humano, ao permitir a apropriação de sistemas de referência do mundo, uma vez que cognição e linguagem são processos imbricados na constituição do sujeito (VYGOTSKY, 2001a).

$O$ processo intersubjetivo fica prejudicado quando há déficits de comunicação, pois na interação um dos sujeitos envolvidos apresentará falhas na construção e na compreensão de sentidos e significados na forma da linguagem expressada. Em tais casos, acredita-se que a Comunicação Alternativa (CA) pode proporcionar subsídios que possam suplementar, complementar ou construir um processo de comunicação (PASSERINO, BEZ, 2013).

No caso, do Transtorno do Espectro Autista (TEA), diversos autores (WALTER 1998; 2000; BEZ; PASSERINO 2009; BEZ 2010; ÁVILA; PASSERINO 2011; ÁVILA 2011; PASSERINO 2012; BEZ; PASSERINO 2012; FOSCARINI;PASSERINO 2012; PASSERINO; BEZ 2013; FOSCARINI 2013) constatam a existência destes déficits de comunicação e utilizam a CA para apoiar o desenvolvimento da comunicação e a consequente interação dos sujeitos com a síndrome.

Nosso grupo de pesquisa TEIAS ${ }^{1}$ tem desenvolvido estudos nesse âmbito desde 2009, a partir de uma visão sócio-histórica cujo foco não está exclusivamente no sujeito, mas na relação deste com o contexto cultural no qual as pessoas estão imersas, constituídas e submetidas às condições de tais contextos (BEZ, 2012).

A partir desta concepção foi desenvolvido o Sistema SCALA2 ${ }^{2}$, composto de um software (tecnologia assistiva) e uma metodologia de uso. O software em si, é um gerenciador de

\footnotetext{
1 Tecnologia na Educação para Inclusão e Aprendizagem em Sociedade da UFRGS.

2 Sistema de Comunicação Alternativa para Letramento de pessoas com Autismo (scala.ufrgs.br/Scalaweb). O Projeto SCALA tem aprovação do comitê de ética da UFRGS.
}

símbolos pictóricos que dão suporte à construção de pranchas e de narrativas visuais (histórias), nas versões web e dispositivo móvel tablet (android).

Neste artigo apresentam-se recortes de relatos das professoras de duas turmas da educação infantil com dois alunos com TEA incluídos no contexto educacional que utilizaram o Sistema Scala com o objetivo de apoiar o desenvolvimento da comunicação no grupo.

\section{Autismo e Comunicação Alterna- tiva (CA)}

O autismo está inserido na categoria diagnóstica dos transtornos de neurodesenvolvimento, em uma categoria específica com o nome de Transtornos do Espectro Autista (TEA), juntamente com o transtorno de Asperger, o transtorno desintegrativo da infância e os transtornos invasivos do desenvolvimento sem outra especificação, conforme a DSM-V (AMERICAN PSYCHIATRIC ASSOCIATION, 2013).

O TEA é um distúrbio do desenvolvimento neurológico presente desde a infância ou do início da infância, em dois domínios: sociais/ comunicação e interesses fixados e comportamentos repetitivos (AMERICAN PSYCHIATRIC ASSOCIATION, 2013).

Os déficits de comunicação no autismo como ausência de intercâmbios corporais expressivos (na comunicação não verbal), falhas nos intercâmbios coloquiais e falas não ajustadas ao contexto (na comunicação verbal) são registrados na literatura (JORDAN; POWELL, 1995). Bosa (2002) em seus estudos descreve sobre a dificuldade inicial de entendimento do que o sujeito com autismo deseja comunicar e sobre o grande esforço que fazem para serem 
compreendidos. Enfatiza ainda que os sujeitos com autismo "[...] não olham porque não sabem a função comunicativa do olhar para compartilhar experiências com as pessoas" (BOSA, 2002, p. 35). Enquanto Goodhart e Baron-Cohen (1993) destacam em suas pesquisas as características especiais do gesto de apontar, Tomasello (2003) foca suas pesquisas na atenção conjunta, considerando que os problemas de comunicação poderiam estar ligados a faIhas na concretização da mesma.

Contrariando alguns autores, segundo os quais, em função do estereótipo, algumas crianças com autismo não são capazes de se comunicar, Molini (2001) identificou, em seus estudos, a presença da intenção comunicativa, mesmo que esta possa ocorrer através de uma forma alternativa de comunicação. O mesmo foi evidenciado na pesquisa de pessoas com autismo realizada por Passerino (2005).

Segundo Passerino (2011), no caso do autismo, os déficits de comunicação podem se manifestar com alterações no uso, forma ou conteúdo da linguagem em nível pragmático e semântico, e em menor medida, em nível sintático, morfossintático, fonológico ou fonético. A importância de utilizar um sistema de Comunicação Alternativa foca-se mais em processos de compreensão e produção de sentidos do que numa produção sonora ou morfossintática.

O uso da Comunicação Alternativa $(C A)^{3}$ com autismo não é recente. Com sujeitos com TEA, as pesquisas iniciam-se com os trabalhos de Walter $(1998 ; 2000)$, que adapta e padro-

\footnotetext{
${ }^{3} \mathrm{~A}$ Comunicação Alternativa é uma da área de investigação inserida no que se conhece como Tecnologia Assistiva (TA), que apoia o desenvolvimento de uma comunicação mais autônoma das pessoas com déficits nesse âmbito. A CA preocupa-se com técnicas, processos e ferramentas que auxiliem a comunicação, como apoio, complementação ou substituição da fala. A importância no uso de CA justifica-se não pelo suporte midiático adotado, mas pelas estratégias e técnicas comunicativas que podem promover a autonomia dos sujeitos em situações de comunicação (BEZ, PASSERINO, VICARI, 2012).
}

niza o sistema PECS 4 para o Brasil. Bez e Passerino (2009); Bez (2010); Ávila e Passerino (2011) e Ávila (2011) apresentam estudos que envolvem o uso de CA com crianças com TEA a partir de propostas metodológicas baseadas numa visão sócio-histórica. Essas pesquisas indicam melhorias significativas nos processos de comunicação dos sujeitos com autismo quando são utilizados sistemas de CA - apoiados por tecnologias digitais - como instrumentos de mediação entre os sujeitos em interação. Desta forma, a comunicação alternativa mostra-se como um fator de facilitação e aproximação ao propiciar outras formas de comunicação e estabelecer, assim, um elo com outras pessoas (AVILA; PASSERINO; REATEGUI, 2012; PASSERINO, 2012; BEZ; PASSERINO, 2012; FOSCARINI; PASSERINO, 2012; PASSERINO; BEZ, 2013; FOSCARINI, 2013).

\section{Sistema SCALA}

Os estudos do grupo iniciam-se em 2009, com o intuito do desenvolvimento do Sistema SCALA que fosse composto de um recurso mais sua metodologia de uso como forma de apoiar o processo de desenvolvimento de pessoas com TEA com déficits de comunicação oral. Inicialmente Bez (2010) utilizou estratégias de mediação com sujeitos com Transtornos Globais do desenvolvimento que deram a base da metodologia hoje empregada no Sistema SCALA. E, seguida iniciou-se o desenvolvimento de um protótipo que foi utilizado por Ávila (2011) com sujeito com autismo. Nossas pesquisas fundamentam-se numa base epistemológica sócio-histórica, portanto para o desenvolvimento da tecnologia foi necessária uma reorganização de conceitos no processo

\footnotetext{
${ }_{4}^{4}$ Picture Exchange Communication System.
} 
de sua construção, pois desejávamos que o mesmo não se fundamentasse apenas na relação sujeito-objeto, mas sim em processos de interação sujeito-objeto-sujeito, na qual o objeto se estabelece como instrumento de mediação ${ }^{5}$. A este processo denominamos Desenvolvimento Centrado em Contextos (DCC) de uso. (PASSERINO; BEZ, 2013; ÁVILA; PASSERINO, 2011; PASSERINO; ÁVILA; BEZ, 2010; BEZ; PASSERINO, 2009).

Portanto, as premissas e a abordagem que norteiam o Sistema Scala contemplam o sujeito em sua individualidade e em interação em diversos contextos com práticas culturais significativas. Desta forma o DCC, contempla os aspectos funcionais dentro do âmbito cultural no qual o individuo está inserido visando, preferencialmente, sua ampliação através de um processo de mediação que vai da aprendizagem ao desenvolvimento humano (VYGOTSKY, 2010).

Cada contexto configura nossas relações e, portanto as mediações possíveis, numa dimensão espaço-temporal, em quatro tipos de tempo: a) o tempo presente, que é o tempo microgenético, esse tempo de agora; b) o tempo vivido, que é o tempo da história enquanto ser humano é o tempo ontogenético; c) o tempo histórico tem aver com a trajetória pessoal de vida de cada indivíduo, relacionado com a cultura; d) o tempo futuro, que é a projeção, expectativas próprias e dos outros, desejos, intenções futuras enquanto ser. Esses quatro tempos atravessam os contextos e se atualizam constantemente e precisam ser levados em conta na construção de uma tecnologia assistiva. (PASSERINO; BEZ, 2013).

\footnotetext{
5 Entende-se mediação, desde uma perspectiva sócio-histórica, como uma cena de atenção conjunta e compartilhada entre dois ou mais sujeitos que utilizam, intencionalmente, instrumentos e signos para promover um processo de apropriação com responsabilidade e competência diferenciadas entre os participantes.
}

O sistema SCALA consiste em um software ${ }^{6}$ em 2 módulos: prancha e narrativas visuais ${ }^{7}$ que permitem, respectivamente a construção de pranchas de comunicação e histórias. Há um conjunto de funcionalidades comuns entre ambos aplicativos tais como importar imagens, editar sons, salvar, exportar, e gerenciar os diferentes arquivos gerados pelo sistema, assim como funcionalidades específicas. O menu à esquerda apresenta ao usuário as categorias de imagens que podem ser utilizadas enquanto a barra horizontal é o menu de trabalho. Partindo de um layout a pessoa pode preencher cada quadro clicando nas categorias de imagens ${ }^{8}$. Cada imagem tem uma legenda padrão que pode ser editada. Para cada quadro é possível também gravar som, ou utilizar o sintetizador de voz inserido no sistema. O sistema prevê a inserção de imagens próprias, permitindo a personalização e adaptação à necessidades de cada contexto. Há também uma funcionalidade de animação das ações que permite a visualização de ações de forma dinâmica (PASSERINO; BEZ, 2013). Nas Figuras 1 e 2 apresentam-se dois exemplos dos módulos prancha e narrativas visuais disponíveis nas plataformas web e dispositivo móvel tablet (sistema operacional Android) ${ }^{9}$.

\footnotetext{
${ }^{6}$ No trabalho nos referiremos ao software somente por SCALA, utilizando a denominação Sistema SCALA quando referenciamos ao conjunto interdependente de programa+metodologia. 7 Desenvolvido sob licença GNU e CreativeCommons para conteúdo aberto. O aplicativo para dispositivos móveis foi desenvolvimento para plataforma Android 3.0 e a versão web, em linguagem PHP5 e Javascript.

${ }^{8}$ Os símbolos pictográficos utilizados são propriedade de CATEDU (http://catedu.es/arasaac/) sob a licença CreativeCommons e foram criados por Sergio Palao.

${ }^{9}$ Explicações detalhadas do sistema podem ser encontradas em: http://scala.ufrgs.br/
} 


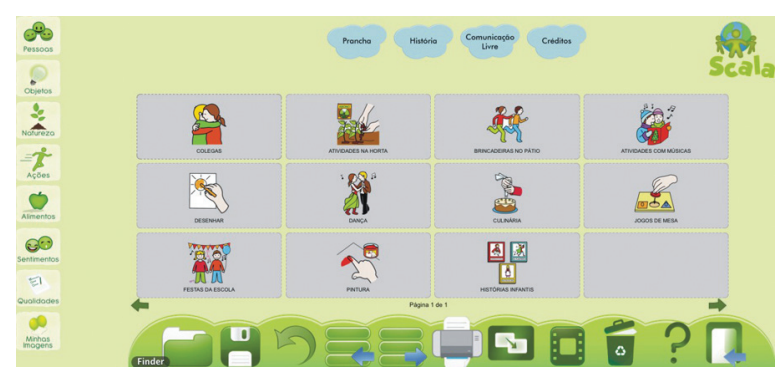

Figura 1. Exemplo do módulo prancha Fonte: Scala.

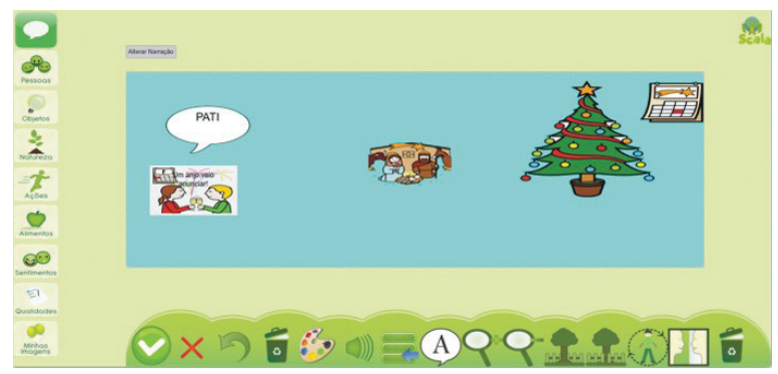

Figura 2. Exemplo do módulo narrativas visuais Fonte: Scala.

O uso do sistema SCALA do ponto de vista metodológico parte de uma perspectiva sócio-histórica na qual o sujeito é inserido em ações mediadoras que potencializam e re-significam a comunicação (PASSERINO, BEZ, 2013). A seguir apresentamos os construtos teóricos que apoiam a análise dos contextos.

\section{A importância do contexto em Bateson e Vygotsky}

Para Bateson (1986, p. 23, destaques do autor), o "[...] 'contexto' está ligado a outra noção indefinida chamada 'significado'. Sem contexto, palavras e ações não têm qualquer significado". O autor estende esta afirmação para todos os tipos de comunicação, tanto a humana (através da palavra escrita, falada, Braille ou sistema gestual como Libras ou mímica) como para todo processo mental, inclusive dos demais seres da natureza - relacionados aos respectivos processos de desenvolvimento, reprodução e sobrevivência.

Ao aprofundar suas reflexões sobre o contexto, Bateson (1986, p. 140), diz que "[...] aprender os contextos da vida é um assunto que tem que ser discutido, não internamente, mas como um assunto de relacionamento externo entre duas criaturas. E o relacionamento é sempre um produto de dupla descrição". Bateson (Idem, p. 142) afirma que há um [...] "aprendizado do contexto" - e "que esse aprendizado do contexto resulta de uma espécie de descrição dupla que acompanha o relacionamento e a interação." Esteves de Vasconcellos (2009, p. 158) explica que, para Bateson, "[...] não é possível descrever e compreender processos relacionais sem descrever os contextos, sem ampliar o foco de observação. Contexto é a palavra necessária na descrição de fenômenos relacionados".

No tocante às relações humanas, o contexto é fundamental ao se pensar a deficiência sob uma perspectiva atual e inclusiva, trazendo uma visão mais abrangente do indivíduo, deixando de encerrar-se na deficiência o critério de acesso (ou não) ao conhecimento, remetendo para a necessidade de vê-lo como parte integrante de uma sociedade que potencialize a sua funcionalidade e participação (CAPITÃO; ALMEIDA, 2011).

O pensamento batesoniano de certa maneira aproxima-se da proposta de Vygotsky (2001a; 2010), que enfatizou a importância do contexto histórico e social, onde o aprendizado se efetiva nas relações entre os pares. O conceito vygotskyano de Zona de Desenvolvimento Proximal (ZDP) - que propõe que aqueles que já dominam determinados conteúdos auxiliem quem ainda precisa de ajuda para a compre- 
ensão de certos conceitos - remete-nos à importância da interrelação, da integração e do respeito entre todos os seres vivos, reflexões que viriam a ser trabalhadas posteriormente por Bateson em sua obra "Mente e Natureza" (1986). Vygotsky (2010, p. 102) destaca que a noção de ZDP propõe uma nova fórmula, a de que "[...] o 'bom aprendizado' é somente aquele que se adianta ao desenvolvimento".

Com as pesquisas empreendidas por Vygotsky e seus colaboradores, houve uma mudança de foco quanto à deficiência - que era estudada sob o ponto de vista clínico, levando à exclusão. Os estudos de Vygotskyressaltaram o potencial a ser desenvolvido no aluno com alguma limitação (supercompensação) e não a deficiência em si (VYGOTSKY, 2001b), o que proporcionou a emergência do paradigma da inclusão em todos os contextos nos quais o indivíduo circula.

Pensando sobre as limitações da mente e do processo mental, Bateson (1986, p. 107, destaques do autor) reflete que como "[...] a mente só pode receber informações de diferenças, existe uma dificuldade em diferençar uma mudança vagarosa e um estado. Existe necessariamente um limite de declive abaixo do qual a inclinação não pode ser percebida". A informação, ou a diferença, salienta Bateson (Ibidem), "[...] sendo da natureza do relacionamento, não está localizada no tempo ou no espaço".

\section{Estudo de Caso: SCALA em con- texto}

O contexto de análise dos recortes de interações deste artigo referem-se ao contexto escolar que ocorreram no ano de 2012. Através dos relatos de quatro professoras de duas turmas de educação infantil com dois alunos (denominados S1 e S2, com idade entre 3 e 5 anos) incluídos com TEA. A descrição desses relatos envolveram processos de mediação com uso do sistema Scala com a tecnologia móvel tablet (Android).

Nos excertos a seguir analisa-se a visão de contexto a partir de Bateson e Vygotsky das interações comunicacionais dos sujeitos foco, as professoras, seus colegas e comunidade escolar.

\section{Excertos da transcrição da filmagem}

A seguir, apresentamos alguns trechos que exemplificam situações vividas pelos sujeitos (S1 e S2) no contexto escolar:

A pesquisadora propõe uma atividade com o SCALA.

Uma professora pergunta se é possível usar imagens da família ou criar historinhas com as crianças.

A Pesquisadora vai explicando para as professoras como incluir histórias no SCALA. P2 pergunta à Pesquisadora se realiza a atividade em conjunto ou separado e a Pesquisadora sugere que seja com toda a turma.

Uma professora pergunta como fazer com que os alunos manipulem o SCALA, Pesquisadora sugere que coloque o SCALA num telão, faz uma roda com os alunos e construindo a história [...].

As professoras sugeriram colocar fotos de um passeio para criar um diálogo interativo, as professoras acreditam que esse exercício sai do concreto para o abstrato.

Como se percebe neste excerto, há entusiasmo por parte das professoras em propor uma atividade de construção colaborativa, em que todos os alunos possam participar, usando como instrumento de mediação o software 
SCALA (ver item 3, Nota Rodapé 4). Importante a capacitação prévia dos professores no uso das tecnologias como ferramentas mediadoras do processo de comunicação, proporcionando apoio à inclusão no contexto da sala de aula e não como um fim em si. A proposta de integrar a turma toda em uma produção coletiva evidencia a importância do contexto trazida por Bateson (1986) - neste caso, o contexto escolar, no qual se evidenciam os relacionamentos entre os sujeitos, ampliando o uso possível de um software - criado originalmente para ser utilizado com crianças que apresentam dificuldades de comunicação - para todos os alunos, sem limitação de tempo ou de espaço para que se configure a informação ou a diferença (BATESON, 1986).

As professoras relatam que houve um evento, na escola, onde S1 surpreendeu desde o primeiro ensaio, ele interagia corretamente com as professoras e colegas. As professoras ficaram surpresas que no dia da apresentação onde tem várias pessoas, o cenário, enfim algo que inibisse o S1, entretanto a apresentação dele foi emocionante.

O aluno S1, com síndrome de autismo, superou suas limitações ao compreender o contexto dos ensaios para a apresentação. Assim, interagiu corretamente com seus colegas e professoras, ou seja, inseriu significado às ações e ao discurso (BATESON, 1986) que se desenrolavam no momento do evento (com cenário e plateia), ou seja, em um outro contexto, diferente daquele vivenciado durante os ensaios - o que nos remete ao que diz $\mathrm{Ba}-$ teson (1986) sobre a importância do contexto para a noção de 'significado'. Complementando esta ideia, o excerto traz bastante claro o que Vygotsky coloca ao dizer que o aprendizado é efetivado nas relações e interações entre os sujeitos, o que nos faz retornar à obra batesoniana, que reforça a importância da inter-relação, da integração e do respeito entre todos os seres vivos. Isto fica bastante claro no excerto, através da avaliação positiva sobre a participação de S1, ou seja, foi valorizado o potencial a ser desenvolvido, tirando o foco da deficiência em si (VYGOTSKY, 2001b).

No primeiro semestre as professoras perceberam grande mudança motora no S1, e agora ele faz o que já está acostumado. As professoras alegam que não estão desafiando ele a pedido da mãe.

Quanto à interação social dele com os colegas, como as professoras têm visto essa questão da comunicação? Elas relatam que o S1 está falando muito mais, está cantarolando mantras, ferramenta utilizada pela fonoaudióloga [...]. O S1 chama alguns colegas pelo nome.

Ele tem pedido as coisas, exemplo, "quebra-cabeça". Elas percebem que ele expressa muito mais, por exemplo, ele grita, briga, ele vai ver o que o colega está fazendo.

Neste excerto, percebe-se, em um primeiro momento, o contexto familiar interferindo no contexto escolar, pois a mãe solicitou às professoras que não desafiassem S1, provocando certa estagnação na aprendizagem do menino. Relembrando Vygotsky (2010), o "bom aprendizado" exige desacomodação e a proposta de constantes desafios ao aluno. Bateson (1986, p. 107 , destaques do autor) refere-se a uma certa dificuldade em estabelecer diferenças "entre uma mudança vagarosa e um estado", por isto a percepção das professoras é de que agora (após a intervenção negativa do contexto familiar), S1 "faz o que já está acostumado". 
A seguir, as professoras relatam crescimento na comunicação oral (cantarola, diz os nomes dos colegas, pede objetos). Pode-se inferir que este seja resultado do trabalho da equipe multidisciplinar, com a utilização de recursos variados, em diversos contextos (os mantras ensinados pela fonoaudióloga, a utilização/mediação do tabletcom o software SCALA (CA)em casa e na escola).

As professoras relatam que S2 progrediu bastante, porque antes ele se agredia, concentração, impulsivo, batia a cabeça, várias estereotipias, S2 está usando medicação, com isso, consegue se acalmar e reduziu as estereotipias, ele abraça os colegas, [está] mais calmo e tem participado mais. [...] Também relatam que melhorou a oralidade, onde numa situação um colega tropeçou e o S2 disse "cuidado" e isso surpreendeu as professoras porque ele usou o termo certo no contexto vivido.

Outra situação surpresa para as professoras: um colega do $\mathrm{S} 2$, de outra turma relatou a professora que ganhou um novo amigo, o S2 onde eles conversaram e brincaram muito. Então, as professoras percebem que S2 está interagindo com os colegas.

Outro fator que pode influenciar determinado contexto onde a criança com autismo circula é o uso de medicação. Neste caso específico, podemos inferir que esta variável foi benéfica, interferindo positivamente no comportamento (acalmou, reduziu as estereotipias de S2). Ao externar sentimento ("cuidado!") em relação ao colega, S2 demonstra afetividade e preocupação em relação ao outro, falando "o termo certo no contexto vivido", ou a dupla descrição decorrente da plena compreensão do contexto, o que amplia o foco da observação, da comunicação e do relacionamento entre os seres (BATESON, 1986). Estes elementos também estão presentes na sua nova amizade com um colega de outra turma, relatado para as professoras, que então perceberam a capacidade de interação que S2 está desenvolvendo, decorrentes da ampliação da suas habilidades comunicativas desenvolvidas no contexto escolar ao longo do tempo histórico, vivido pelo sujeito (BEZ, 2012). Pois a informação (ou a diferença) "[...] sendo da natureza do relacionamento, não está localizada no tempo ou no espaço" (BATESON, 1986, p. 107).

\section{Considerações finais}

A partir do presente estudo percebe-se que o uso do SCALA mostra-se potencializador como instrumento mediador de ações comunicativas com turmas nas quais participam sujeitos com TEA.

As interações foram potencializadas a partir da utilização do sistema e foi possível perceber, como afirma Bateson (1986), algumas mudanças sutis que evidenciam movimentos e processos que podem passar despercebidos em situações cotidianas. A importância de pesquisas deste tipo mostra a viabilidade do uso de um sistema de CA para apoiar a inclusão e metodologicamente apresenta possibilidades de práticas educativas contextualizadas. Há, contudo, necessidade de aumentar pesquisas nesse âmbito do uso da CA nos processos inclusivos. 


\section{Referências}

AMERICAN PSYCHIATRIC ASSOCIATION. Diagnostic and Statistical Manual of Mental Disorders: DSM-5.5. ed. Washington, DC: APA,2013.

AVILA, B. G. Comunicação Aumentativa e Alternativa para o Desenvolvimento da Oralidade de Pessoas com Autismo. Porto Alegre, 2011. 168 f + Apêndices. Dissertação (Mestrado) - Programa de Pós-Graduação em Educação, Faculdade de Educação, Universidade Federal do Rio Grande do Sul, Porto Alegre, 2011. Disponível em:

<http://www.lume.ufrgs.br/bitstream/handle/10183/32307/000785427.pdf?sequence=1>. Acesso em: 15 mar. 2013.

AVILA, B. G; PASSERINO, L. M. Comunicação Aumentativa e Alternativa e Autismo: desenvolvendo estratégias por meio do SCALA. In: SEMINÁRIO NACIONAL DE PESQUISA EM EDUCAÇÃO Práticas Pedagógicas na educação especial: multiplicidade do atendimento educacional especializado, 6 . Anais... Nova Almeida da Serra, ES, 2011 v. 1. P. 1-10.

AVILA, B. G.; PASSERINO, L. M.; REATEGUI, E. Proposta de uma Metodologia para a Construção de um Sistema de CAA Focado no Contexto de seus Usuários. Revista Brasileira de Informática na Educação, v. 20, p. 87-96, 2012.

BATESON, G. Mente e Natureza. Rio de Janeiro: Francisco Alves, 1986.

BEZ, R. M. Comunicação Aumentativa e Alternativa para Sujeitos com Transtornos Globais de Desenvolvimento na Promoção da Expressão e Intencionalidade por Meio de Ações Mediadoras. 2010. Dissertação (Mestrado) - Programa de Pós-Graduação em Educação, Faculdade de Educação, Universidade Federal do Rio Grande do Sul, Porto Alegre, 2010. Disponível em:

<http://www.lume.ufrgs.br/bitstream/handle/10183/26303/000757639.pdf?sequence=1>. Acesso em: 18 mar. 2013.

BEZ, M. R.; PASSERINO, L. M. Applying Alternative and Augmentative Communication to an Inclusive Group. In: TATNALL, A.; JONES, A. (Ed.). IFIP WORLD CONFERENCE ON COMPUTERS IN EDUCATION:WCCE, 9., 2009, Bento Gonçalves. Proceedings... Bento Gonçalves, IFIP WCCE, 2009. V. 1, p. 164-174.

BEZ, M. R.; PASSERINO, L. M.; VICARI R. M. Scalando em Tablets: comunicação alternativa em foco. Educação e Contemporaneidade: contextos e singularidades. Bahia: EDUFBA, 2012.

BOSA, C. Atenção compartilhada e identificação precoce do autismo. Psicologia: Reflexão e Crítica, Porto Alegre, v. 15, p. 77-88, 2002. 
CAPITÃO, S.; ALMEIDA, A. O Uso das TIC para a Inclusão dos Alunos com Necessidades Educativas Especiais. Indagatio Didactica, Portugal, v. 3, n. 2, p. 56-67, jun. 2011.

eSTeVes De VASCONCELlOS, M. J. Pensamento Sistêmico: o novo paradigma da ciência. 8. ed. Campinas, SP: Papirus, 2009.

FOSCARINI, Ana Carla. A Intencionalidade de Comunicação Mediada em Autismo: um estudo de aquisição de gestos no sistema SCALA. 2013. Dissertação (Mestrado em Educação) - Faculdade de Educação, Programa de Pós-Graduação em Educação, Universidade Federal do Rio Grande do Sul, Porto Alegre, 2013.

FOSCARINI, A. C.; PASSERINO, L. M. Mediação e Desenvolvimento no Atendimento Educacional Especializado Através do Uso de Artefatos Tecnológicos. ANPED SUL SEMINÁRIO DE PESQUISA EM EDUCAÇÃODA REGIÃo SUL: A Pós-Graduação e Suas Interlocuções com a Educação Básica, 10, 2012, Caxias do Sul, RS. Anais... Caxias do Sul: ANPED, 2012. V. 1, p. 1-15.

GOODHART, F.; BARON-COHEN, S. How Many Ways Can the Point be Made? Evidence from children with and without autism. First Language, 13, 1993, p. 225-233.

JORDAN, R.; POWELL, S. Understanding and Teaching Children with Autism. West Sussex, England: John Wiley\&Sons, 1995.

MOLINI, D. R. Verificação de Diferentes Modelos de Coleta de Dados dos Aspectos Sociocognitivos na Terapia Fonoaudiológica de Crianças com Distúrbios Psiquiátricos. 2001. 230f. Dissertação (Mestrado em Ciências) - Universidade de São Paulo, São Paulo, 2001.

PASSERINO, L. M. Pessoas com Autismo em Ambientes Digitais de Aprendizagem: estudo dos processos de interação social e mediação. 315 f. Tese (Doutorado) - Universidade Federal do Rio Grande do Sul. Faculdade de Educação. Programa de Pós-Graduação em InformáticanaEducação, Porto Alegre, 2005. Disponível em:

<http://capesdw.capes.gov.br/capesdw/resumo.html?idtese=20054542001013075P9>. Acesso em: 16 maio 2013.

PASSERINO, L. M. Salas de Recursos, Tecnologia Assistiva e Processos de Inclusão a Partir da Perspectiva Sócio-histórica. In: MORAIS, S. C. de (Org.). Educação Inclusiva: diferentes significados. Porto Alegre: Avangraf, 2011. P. 67-77.

PASSERINO, L. M.; AVILA, B. G.; BEZ, M. R. SCALA: um Sistema de Comunicação Alternativa para o Letramento de Pessoas com Autismo. RENOTE: Revista Novas Tecnologias na Educação, v. 1, p. 1-10, 2010. 
PASSERINO, L. M.; BEZ, M. R. Building an Alternative Communication System for Literacy of Children with Autism (SCALA) with Context-Centered Design of Usage. Autism: Book 1. InTech, 2013.

TOMASEllo, M. Origens Culturais da Aquisição do Conhecimento Humano. São Paulo: Martins Fontes, 2003.

VYGOTSKY, L. S. A Formação Social da Mente: o desenvolvimento dos processos psicológicos superiores. 7. ed. São Paulo: Martins Fontes, 2010.

A Construção do Pensamento e da Linguagem. São Paulo: Martins Fontes, 2001.

Obras Escogidas: fundamentos de defectología. 2. ed. Madrid: Visor, 1997-2001. V. 5.

WALTER, C. AAdaptação do Sistema PECS de Comunicação para o Brasil: uma comunicação alternativa para pessoas com autismo infantil. In: MARQUEZINE, M. C, ALMEIDA, M. A.; TANAKA E.; MORI N., SHIMAZAKI, E. (Org.) Perspectivas Multidiciplinares em Educação Especial. Londrina: UEL, 1998. P.277-280.

WALTER, C. Os Efeitos da Adaptação do PECS Associada ao Curriculum Funcional em Pessoas com Autismo Infantil. 2000. Dissertação (Mestrado) - Universidade Federal de São Carlos, São Carlos, SP, 2000.

Submetido para avaliação em 04 de novembro de 2013.

Aprovado para publicação em 20 de dezembro de 2013.

Katia Soares Coutinho: Técnico Científico na Bibliotecária da Escola Estadual Técnica em Saúde do Hospital de Clínicas de Porto Alegre - Porto Alegre - RS - Brasil.

E-mail: katias_coutinho@hotmail.com

Maria Rosangela Bez: Doutoranda do Programa de Pós-Graduação em Informática na Educação da Universidade Federal do Rio Grande do Sul - Porto Alegre - RS - Brasil. E-mail: bezrosangela@gmail.com

Liliana Maria Passerino: Professora adjunta da Universidade Federal do Rio Grande do Sul - Porto Alegre - RS Brasil. E-mail: liliana@cinted.ufrgs.br 